\title{
Problems in Communication Between Heavy Flavor Experiment and Hadron Spectroscopy
}

\author{
Harry J. Lipkin ${ }^{1}$ \\ Department of Particle Physics Weizmann Institute of Science, Rehovot 76100, Istae \\ School of Physics and Astronomy, Raymond and Beverly Sackler Faculty of Exact \\ Sciences, Tel Aviv University, Tel Aviv, Israel

High Energy Physics Division, Argonne National Laboratory, Argonne, IL 60439-4815, $U S A$

\begin{abstract}
The implications of hadron spectroscopy are generally overlooked both by experimenters presenting results and theorists making predictions. Two examples are (1) possible effects of nodes in wave functions of final state mesons produced in $B$ decay; (2)Dependence of predictions for $B$ decays into final states containing $\eta$ or $\eta^{\prime}$ on models and mixing angles for these states.
\end{abstract}

The remarks about Carl Dover in this session dedicated to his memory here are brief. The full International Conference on Hypernuclear and Strange Particle Physics (HYP97) at Brookhaven, Oct.13-18, 1997 was devoted to his memory. Carl made outstanding contributions to hadron spectroscopy before he was taken from us much too soon. I had the good fortune to collaborate with him in trying to understand the $H$ dibaryon [1], We encountered problems in communication between experimenters, theorists, and lattice calculators. Interesting physics underlying the result in one lattice work [2] that the $H$ was unbound was not mentioned in the paper but revealed only by private communication [3]. Six quarks starting at the same point on the lattice dissociated into two $\Lambda$ 's kept apart by repulsive quark exchange. The lattice technologists cared only about the reliability of their small lattice calculation. The existence of this previously ignored repulsive interaction was lost in the published paper even though it was later considered to be sufficiently impor-

1) Supported in part by grant No. I-0304-120-.07/93 from The German-Israeli Foundation for Scientific Research and Development and by the U.S. Department of Energy, Division of High Energy Physics, Contract W-31-109-ENG-38. 


\section{DISCLAIMER}

This report was prepared as an account of work sponsored by an agency of the United States Government. Neither the United States Government nor any agency thereof, nor any of their employees, make any warranty, express or implied, or assumes any legal liability or responsibility for the accuracy, completeness, or usefulness of any information, apparatus, product, or process disclosed, or represents that its use would not infringe privately owned rights. Reference herein to any specific commercial product, process, or service by trade name, trademark, manufacturer, or otherwise does not necessarily constitute or imply its

endorsement, recommendation, or favoring by the United States Government or any agency thereof. The views and opinions of authors expressed herein do not necessarily state or reflect those of the United States Government or any agency thereof. 


\section{DISCLAIMER}

Portions of this document may be illegible in electronic image products. Images are produced from the best available original document. 
tant to be considered by non-lattice theorists and experimenters. Fortunately this information was extracted by personal contacts $[3$ and its implications were discussed with Carl and others and led to interesting conclusions $[1,13]$

We miss Carl, and his works will continue to remind us of him.

Decays of heavy flavor hadrons into light hadrons can be sensitive to details of hadron spectroscopy, particularly when the final states contain excited state mesons with nodes in the wave functions [5] or flavor mixed neutral mesons like the $\eta$ and $\eta^{\prime}[6-8]$. This interface has unfortunately not been given sufficient attention at this conference. We note two factors responsible for this communication breakdown which should be remembered in organizing future meetings.

One problem arises because the relevant data in heavy quark physics generally come from large collaborations. The question of who should present the results and future programs for these collaborations is nontrivial. The present policy seems to be that the collaborations choose for themselves the participants in a given conference, rather than to allow the conference organizers to choose speakers who have a broad view of the area and can give good talks. It is understandable that data should be presented by the young physicists like postdocs, who have done the work and are very familiar with its details and the reliability of the numbers quoted. It is also understandable that postdocs must be able to establish a record to enable them to compete on the job market. However, in an interdisciplinary area like hadron spectroscopy, it is important also to have participants familiar with this area.

One example at Hadron97 was the presentation of results from CLEO on $B$ decays to final states containing the $\eta$ and $\eta^{\prime}$. The speaker quoted the agreement between the data and theoretical predictions, but was unable to tell the audience when asked which model for the $\eta$ and $\eta^{\prime}$, which wave functions and which mixing angles were used in the predictions. This is of particular interest at a hadron spectroscopy conference, because recent data [9] on the decays $B \rightarrow K \eta$ and $B \rightarrow K \eta^{\prime}$ suggest that the standard treatment cannot predict the large $K \eta^{\prime}$ decay. There have therefore been suggestions [10] that intrinsic charm or glueballs may be present. A serious discussion [6-8] of this point in view of the latest data would have been very useful.

The second problem is the failure of the $B$-decay theoretical establishment to recognize the relevance of hadron physics and hadron spectroscopy $[11,12]$. The surprising enhancement of $B^{ \pm}$decays to low-lying exclusive channels was first noted and explained with a hadron spectroscopy approach [5]. It confounded the theoretical establishment by requiring constructive interference between color suppressed and color favored contributions, in contrast with previous predictions [11]. Furthermore the enhancement could not be general because the $B^{\circ}$ and $B^{ \pm}$lifetimes are nearly equal. There must therefore be other exclusive channels where the interference is reversed, so that it cancels out in the total width. Recently the conventional description [11] has been modified $[12$ to include an additional energy-dependent (fudge?) factor that 
explains the enhancement and insists that the same enhancement should hold in $B \rightarrow D a_{1}$ and other quasi-two-body final states independently of the hadron spectroscopy. But this modified description gives no clue to the identity of the final states where the interference is reversed, and the assumption [11] that standard model B-decay is well understood and described by calculating the right diagrams has been questioned $[5,13]$.

In the hadron spectroscopy approach [5] the final states where constructive interference is observed are seen to all have nodeless s-wave quark-model wave functions. The relative phase of the suppressed and favored diagrams depends upon hadron form factors whose signs can be reversed by the presence of nodes or orbital angular momentum in the wave functions. This wave function dependence can solve the problem of equal lifetimes and be checked experimentally by looking for systematic differences in the interference in final states containing excited quark-model wave functions in both $B$ and $D$ decays; e.g. $B \rightarrow D a_{1}$. Present data are still inconclusive, but the next round of CLEO data is expected to hopefully resolve this point [14]. However there was no discussion of this issue at Hadron97 following the experimental reports from CLEO which dismissed this point as "now explained by the standard theory." That the role of hadron spectroscopy is at least controversial and that it is supported by two members of the International Advisory Committee for this conference is an indication that it deserves discussion.

The questions of how weak decays of charmed mesons may be influenced by the presence of known meson resonances in this mass region [15] and possible experimental investigations have also not been considered.

\section{REFERENCES}

1. Murray A. Moinester, Carl B. Dover and Harry J. Lipkin, Phys. Rev. C46 (1992) 1082

2. P. MacKenzie and H. Thacker, Phys. Rev. Letters 65, 2539 (1985)

3. H. Thacker, private communication

4. Harry J. Lipkin, Nuclear Physics A in press

5. Frank E. Close and Harry J. Lipkin, Physics Letters B405 (1997) 157

6. Harry J. Lipkin, Phys. Lett. in press

7. Harry J. Lipkin, Physics Letters B254 (1991) 247

8. Harry J. Lipkin, Phys. Lett. B357 (1995) 404

9. B. Behrens, [CLEO] talk at $B$ Physics and CP Violation, Waikiki, HI (March 1997)

10. D. Atwood and A. Soni, Phys. Lett. B405 (1997) 150

11. M. Bauer, B. Stech and M. Wirbel, Z. Phys. C 34 (1987) 103

12. B. Stech talk at $B$ Physics and CP Violation, Waikiki, HI (March 1997)

13. Harry J. Lipkin, Nuclear Physics A in press

14. T. Browder, private communication

15. Frank E. Close and Harry J. Lipkin, Phys. Lett. B372 (1996) 306 
From: SMTP\%"FTLIPKIN@wiswic.weizmann.ac.il" 18-NOV-1997 12:45:33.91

To: GOTLUND

CC:

Subj: I hope that this is satisfactory.

Date: Tue, 18 Nov 1997 20:46:17 GMT

From: FTLIPKIN@wiswic.weizmann.ac.il

To: GOTLUND@HEP.ANL.GOV

CC: ELB@HEP.ANL.GOV, HJL@AXP1.HEP.ANL.GOV

Message-Id: <971118204617.21e00e3b@wiswic.weizmann.ac.il>

Subject: I hope that this is satisfactory.

Hi Sandy,

I hereby certify that the two conference papers ("Problems

at the Interface Between Heavy Flavor Physics, QCD and Hadron Spectroscopy" and

"Problems in Communication Between Heavy Flavor Experiment and Hadron Spectroscopy")

1. Disclose no invention or copyrightable software to the best of my knowledge,

2. Do not include information covered by a nondisclosure agreement to protect the interests of either ANL or an outside organization,

3. Do not include information required to be protected by a Cooperative Research and Development Agreement, Pilot Center Agreement, or other Laboratory agreement.

Sincerely yours,

Harry J. Lipkin 